Jeremy B M Jowett; Yasunori Okada; Peter J Leedman; Joanne E Curran; Matthew P Johnson; Eric K Moses; Harald H H Goring; Satsuki Mochizuki; John Blangero; Leah Stone; Holly Allen; Chris Mitchell; Vance B Matthew, ADAM28 is elevated in humans with the metabolic syndrome and is a novel sheddase of human tumour necrosis factor-a. Immunology and Cell Biology 2012, 90 (10), p. 966-973 doi: 10.1038/icb.2012.44

(C) 2013 Australasian Society for Immunology

This is pre-copy-editing, author-produced version of an article accepted for publication in Immunlogy and Cell Biology following peer review. The definitive published version (see citation above) is located at the publisher's site.

This version was made available in the UWA Research Repository on 13 August 2013 in compliance with the publisher's, Nature Publishing Group, policies on archiving in institutional repositories.

Use of the article is subject to copyright law. See reprints \& permissions@npg 


\section{ADAM28 is elevated in humans with the metabolic syndrome and is a novel sheddase of human tumour necrosis factor- $\alpha$.}

Jeremy B. M. Jowett ${ }^{1 *}$, Yasunori Okada ${ }^{1 \dagger}$, Peter J. Leedman ${ }^{\ddagger}$, Joanne E. Curran॥, Matthew P. Johnson\|, Eric K. Moses॥, Harald H.H. Goring\|, Satsuki Mochizuki ${ }^{\dagger}$, John Blangero", Leah Stone", Holly Allen ${ }^{\ddagger}$, Chris Mitchell ${ }^{\ddagger}$, Vance B. Matthews $\$$.

*Baker IDI Heart \& Diabetes Institute, 75 Commercial Road, Melbourne, Victoria, 3004, Australia. $\dagger$ Department of Pathology, School of Medicine, Keio University, 35 Shinanomachi, Shinjuku-ku, Tokyo, 160-8582, Japan.

†UWA Centre for Medical Research, Western Australian Institute for Medical Research, Level 6, MRF Building, Rear 50 Murray Street, Perth, Western Australia, 6000, Australia.

\#School of Medicine and Pharmacology, The University of Western Australia, 35 Stirling Highway, Crawley, Perth, Western Australia, 6009, Australia.

\|Texas Biomedical Research Institute, PO Box 760549, San Antonio, Texas, 78227-5301, USA.

${ }^{1}$ These authors contributed equally.

Running head: ADAM28 is elevated with the metabolic syndrome and sheds TNF- $\alpha$

Keywords: metalloproteinase, metabolism, cytokines, insulin resistance.

Abbreviations:

ADAM: A disintegrin and metalloproteinase

HEK: human embryonic kidney

IGFBP: insulin-like growth factor binding protein-3

IKK: IאB kinase

JNK: c-Jun terminal amino kinase

LPS: lipopolysaccharide

MMP: matrix metalloproteinase

PSGL-1: P-selectin glycoprotein ligand-1

PTP: protein tyrosine phosphatases

SAFHS: San Antonio Family Heart Study

siRNA: Small interfering RNA

TACE: TNF- $\alpha$ converting enzyme 
TIMP: tissue inhibitor of metalloproteinase

TNF- $\alpha$ : tumour necrosis factor- $\alpha$

T2D: Type 2 diabetes.

Address for correspondence:

Vance B. Matthews

Western Australian Institute for Medical Research,

Level 6, MRF Building, Rear 50 Murray Street,

Perth, WA, 6000, Australia.

Ph: 61892240333 Fax: 61892240322

email: vance.matthews@waimr.uwa.edu.au

Abstract: 201 words Text: 4025 words 


\begin{abstract}
Metalloproteinases are implicated in cleaving numerous pro-inflammatory mediators from the cell surface. Interestingly, elevated levels of TNF- $\alpha$ have been associated with the metabolic syndrome. We aimed to ascertain whether the human metalloproteinase ADAM28 correlates with parameters of the metabolic syndrome and if ADAM28 is a novel sheddase of human TNF- $\alpha$. To identify novel metalloproteinases associated with the metabolic syndrome, we conducted micro-array studies on peripheral blood mononuclear cells from a well characterised human cohort. Human ADAM28 and TNF- $\alpha$ were over-expressed and ADAM28 expression or activity was reduced with siRNA or pharmacological inhibition. TNF- $\alpha$ levels were measured in cell supernatant by ELISA. We also conducted ADAM28 inhibition studies in human THP1 macrophages. Human ADAM28 expression levels were positively correlated with parameters of the metabolic syndrome. When human ADAM28 and TNF- $\alpha$ were over-expressed in HEK293 cells, both proteins co-localised, coimmunoprecipitated and promoted TNF- $\alpha$ shedding. The shedding was significantly reduced when ADAM28 activity was inhibited or ADAM28 expression was down-regulated. In human THP-1 macrophages, endogenous ADAM28 and TNF- $\alpha$ were co-expressed and TNF- $\alpha$ shedding was significantly reduced when ADAM28 was inhibited by pharmacological inhibition or siRNA knock-down. Our data suggests a novel mechanistic role for the metalloproteinase ADAM28 in inflammation, obesity and type 2 diabetes.
\end{abstract}




\section{INTRODUCTION}

The prevalence of adult obesity has increased approximately $75 \%$ in the last quarter century with an alarming increase in overweight and obese children in both developed and developing countries ${ }^{1}$. An abundant number of disorders directly correlate with obesity. These include a number of cancers, glucose intolerance, dyslipidemia, cardiovascular disease and insulin resistance which may ultimately culminate in pancreatic beta cell failure and type 2 diabetes (T2D). The understanding of novel mechanisms underlying the pathogenesis of $\mathrm{T} 2 \mathrm{D}$ and the development of new strategies to treat 'metabolic disease' is most warranted.

Growing evidence suggests that during T2D, a state of chronic inflammation exists in metabolically active tissues such as the liver, adipose tissue and skeletal muscle. A number of studies have elegantly demonstrated the critical role that inflammatory cascades play in the development of obesity and T2D. Serine threonine kinases such as c-Jun terminal amino kinase (JNK) or IKB kinase $\beta / \varepsilon$ (IKK- $\beta / \varepsilon$ ) and protein tyrosine phosphatases (PTP) such as PTP1B which attenuate insulin signaling, may be activated by the production of fatty acid metabolites within insulin responsive tissues $^{2,3,4,5,6}$.

Soluble pro-inflammatory cytokines including interleukin-6 (IL-6) and granulocyte macrophage colony stimulating factor have exhibited profound beneficial metabolic effects. These include promotion of fat oxidation and insulin sensitivity and reductions in food intake and body weight respectively ${ }^{7,8}$. The adipokines, adiponectin and leptin may also have a positive effect on body weight regulation ${ }^{9,10}$. Additionally, over-expression of interleukin-10 protected mice from dietinduced inflammation and insulin resistance in skeletal muscle ${ }^{11}$. In contrast, a vast amount of research has implicated a number of soluble factors such as tumour necrosis factor- $\alpha$ (TNF- $\alpha)$ in the etiology of obesity and insulin resistance in rodents and humans ${ }^{12,13,14,15,16}$. However, it should be highlighted that numerous pro-inflammatory cytokines may work in concert to promote an insulin resistant phenotype in metabolic cell types.

Cell membrane TNF- $\alpha$ may be cleaved by the metalloproteinase TNF- $\alpha$ converting enzyme (TACE), otherwise known as ADAM17, a member of a disintegrin and metalloproteinase (ADAM) gene family. Another metalloproteinase, ADAM28 is expressed in numerous cell types including lymphoid cells, and has two isoforms: (i) a membrane-type form (ADAM28m) and (ii) a secreted soluble form (ADAM28s). Our previous study demonstrated that the $65 \mathrm{kD}$ pro-ADAM28 is processed by matrix metalloproteinase-7 (MMP-7) to 40 and $42 \mathrm{kDa}$ forms which results in an 
active form of ADAM $28^{17}$. We and others have previously identified a number of novel substrates for ADAM28 which include insulin-like growth factor binding protein-3 (IGFBP-3), connective tissue growth factor, proteoglycans, myelin basic protein and CD23 $3^{17,18,19,20,21}$. Interestingly, a number of synthetic peptides containing the authentic TNF- $\alpha$ shedding site were shown to be cleaved by ADAM $28^{21}$. This was a very exciting finding which reinforced the notion that ADAM28 may be a novel sheddase of one of the major pro-inflammatory cytokines involved with the metabolic syndrome.

In this study, we hypothesised that ADAM28 is associated with obesity and T2D in a human cohort and that ADAM28 may be a novel sheddase of TNF- $\alpha$ which may promote metabolic dysfunction. The outcomes of this study will provide new insights into the metalloproteinase mediated pathogenesis of obesity and T2D.

\section{RESULTS}

ADAM28 expression in human blood mononuclear cells is a novel marker of the metabolic syndrome

Here we use a large human cohort well characterised for T2D and cardiovascular disease that has also been extensively genetically analysed and carries full genome wide gene expression profiles on 1,240 individuals. This set was filtered to identify novel metalloproteinases involved in the development of obesity and type 2 diabetes. We have found that high-level expression of ADAM28 in blood mononuclear cells from the San Antonio Family Heart Study (SAFHS) cohort correlated strongly with parameters of the metabolic syndrome and in particular BMI and relative fat (Table 1). These highly significant clinically relevant observations suggest a role for ADAM28 in the regulation of human metabolism.

\section{ADAM28 is a novel sheddase of the pro-inflammatory cytokine TNF- $\alpha$ in HEK293 cells}

Numerous studies have demonstrated that levels of circulating TNF- $\alpha$ positively correlate with body mass index. Prior reports have also suggested that ADAM28 is able to cleave synthetic peptides possessing the TNF- $\alpha$ shedding site ${ }^{21}$. In initial experiments, we tested the hypothesis that ADAM28 may be a novel sheddase of cell membrane TNF- $\alpha$ protein. When human ADAM28 and TNF- $\alpha$ were co-expressed in human HEK-293 cells, both proteins co-localised (a critical requirement for shedding) (Figure 1A-C). In addition, co-immunoprecipitation experiments in these same cells highlighted that human ADAM28 co-precipitated with the $27 \mathrm{kD}$ membrane bound proTNF- $\alpha$ (Figure 1D). 
Significantly, co-transfection of human TNF- $\alpha$ with human ADAM28 expression vectors in HEK293 cells promoted TNF- $\alpha$ shedding four-fold (Figure 2A). We previously demonstrated that the synthetic inhibitor KB-R7785 significantly reduced ADAM28 activity. When the ADAM28 inhibitor was added to HEK293 cells co-transfected with human TNF- $\alpha$ and human ADAM28 expression vectors, we were able to reduce the levels of soluble TNF- $\alpha$ in the cell-free culture supernatant to the basal levels which existed when the TNF- $\alpha$ vector was transfected alone (Figure 2B). Immunoblotting of the cell lysates of cells co-transfected with human TNF- $\alpha$ and human ADAM28 expression vectors with and without KB-R7785 also showed that there were markedly lower levels of mature shed TNF- $\alpha(17 \mathrm{kD})$ when the ADAM28 inhibitor was added. This also supports that the shed TNF- $\alpha$ may bind the TNF- $\alpha$ receptor on the cell surface of the HEK293 cells (Figure 2C) which is of critical importance for later experiments (Figure 4A and B). These data provide compelling evidence that ADAM28 is a major metalloproteinase involved in TNF- $\alpha$ cleavage, however we could not exclude the possibility that ADAMs other than ADAM28 also play a role in TNF- $\alpha$ shedding in the transfectants. As molecular knock-down of ADAM28 should provide definitive evidence of its role in TNF- $\alpha$ shedding, we conducted ADAM28 siRNA experiments in a HEK293 over-expression system where all cells were transfected with human TNF- $\alpha$ and human ADAM-28 expression vectors. We chose to use a scrambled Cy3 labelled siRNA as our negative control. This indicated that we were able to obtain greater than $90 \%$ transfection efficiency. We also used three different siRNA sequences directed at human ADAM28 mRNA. Importantly, all three ADAM28 siRNAs were able to significantly down-regulate the ADAM28 mRNA to less than one quarter of those levels observed for the scrambled siRNA control (Figure $3 \mathrm{~A})$. Additionally, both the pro-form $(62 \mathrm{kD})$ and the active form $(42 \mathrm{kD})$ of the human ADAM28 protein were significantly reduced with all three siRNAs (Figure 3B, C and D). As the active form of ADAM28 was also reduced to less than one quarter of those levels observed for the scrambled siRNA control (Figure 3D), we sought to determine whether the level of TNF- $\alpha$ shedding was also reduced with ADAM28 siRNAs. All three ADAM28 siRNAs significantly reduced TNF- $\alpha$ cleavage compared to the scrambled siRNA control (Figure 3E; top panel). Importantly, the cell membrane bound form of TNF- $\alpha$ (pro-TNF- $\alpha$ ) was elevated in ADAM28 siRNA transfected cells compared to cells transfected with scrambled siRNA (Figure 3E; bottom panel).

\section{ADAM28 cleaved TNF- $\alpha$ is bioactive and up-regulates ADAM28 protein levels.}


To ascertain whether the ADAM28 cleaved TNF- $\alpha$ was bioactive, we analysed critical signalling pathways downstream of TNF- $\alpha$ binding. We examined phosphorylation of c-Jun N-terminal kinase (JNK). Interestingly, the cellular protein lysates from HEK293 cells transfected with both TNF- $\alpha$ and ADAM28 expression vectors displayed a considerable elevation in JNK phosphorylation suggesting that the elevated TNF- $\alpha$ protein was bioactive (Figure $4 \mathrm{~A}$ and $\mathrm{B}$ ). In an effort to determine whether the TNF- $\alpha$ shedding by ADAM28 may result in inflammation in metabolically relevant cell types, we continued with HEK293 conditioned media experiments in L6 skeletal myotubes. When we treated L6 myotubes with either cell free conditioned media from HEK293 cells transfected with either empty and TNF- $\alpha$ vectors (TNF- $\alpha$ ) or TNF- $\alpha$ and ADAM28 vectors (TNF- $\alpha /$ ADAM28), we observed significantly elevated phosphorylation of JNK when cells were treated with TNF- $\alpha$ /ADAM28 conditioned media (Figure 4C and D).

We next aimed to determine whether ADAM28-mediated TNF- $\alpha$ release may instigate a positive feedback loop whereby ADAM28 levels are elevated with TNF- $\alpha$ stimulation. Using an ADAM28 ELISA, we demonstrated that ADAM28 levels were increased approximately four-fold when TNF$\alpha$ and ADAM28 vectors were co-transfected ( $2 \mu \mathrm{g}$ each) compared to when ADAM28 and empty vectors were co-transfected ( $2 \mu \mathrm{g}$ each) into HEK293 cells (Figure 5A). This eludes to a mechanism by which ADAM28-mediated TNF- $\alpha$ shedding may be maintained. We also conducted TNF- $\alpha$ stimulation experiments in THP1 monocytes and assessed endogenous human ADAM28 mRNA levels. Consistent with the HEK293 studies, TNF- $\alpha$ stimulation resulted in elevated ADAM28 mRNA levels (Figure 5B).

\section{Inhibition of ADAM28 activity inhibits endogenous TNF- $\alpha$ shedding in PMA-induced human macrophages and LPS stimulated human monocytes.}

It has previously been reported that treatment of human THP-1 monocytes with PMA results in differentiation to macrophages and increases expression of ADAM28 mRNA after 24hrs of treatment. As we were interested in upregulating ADAM28 and TNF- $\alpha$ protein expression, we treated THP-1 monocytes with PMA for $48 \mathrm{hrs}$. We definitively demonstrated that the cells became adherent and displayed characteristic macrophage morphology. Using this established human macrophage cell culture model in which endogenous ADAM28 (Figure 6A) and TNF- $\alpha$ (Figure 6B) are co-expressed, we then aimed to determine whether inhibition of ADAM28 activity with the pharmacological inhibitor, KB-R7785, may influence TNF- $\alpha$ shedding. Consistent with our experiments in HEK293 cells, when we treated THP-1 differentiated macrophages with KB-R7785, 
TNF- $\alpha$ shedding was significantly reduced (Figure 6B). As we have previously shown that we may successfully knock-down ADAM28 expression utilising siRNA technology (Figure 3), we next aimed to utilise siRNA to reduce endogenous ADAM28 expression in THP1 monocytes. We were able to reduce ADAM28 expression levels by at least $50 \%$. To promote TNF- $\alpha$ expression in THP1 monocytes, we stimulated cells with $1 \mu \mathrm{g} / \mathrm{ml}$ lipopolysaccharide for $48 \mathrm{hrs}$. In agreement with our previous findings, we now demonstrate that shedding of endogenously expressed human TNF- $\alpha$ may be significantly reduced by knock-down of endogenously expressed human ADAM28 (Figure $6 \mathrm{C})$.

\section{DISCUSSION}

We have shown for the first time that ADAM28 expression levels in peripheral blood mononuclear cells isolated from a large human cohort are strongly correlated with parameters of the metabolic syndrome and that ADAM28 promotes the shedding of the cytokine TNF- $\alpha$, which has been implicated in the metabolic syndrome.

An obesogenic role for the metalloproteinase TACE has been concluded from knock-out mouse studies. Interestingly, heterozygous TACE KO mice are significantly protected from obesity and insulin resistance ${ }^{22}$. Conversely, ablation of tissue inhibitor of metalloproteinases 3 (Timp3), which is the physiological inhibitor of TACE, promotes hyperglycemia, hyperinsulinemia, glucose intolerance and insulin resistance in mice fed a high fat $\operatorname{diet}^{23}$. Furthermore, it appears hyperglycemia may be the stimulus for elevated TACE expression in obesity as exposure of aortic smooth muscle cells to high glucose increased TACE activity and promoted shedding of TNF- $\alpha$ via protein kinase $\mathrm{C}-\delta^{24}$. TACE is currently considered a therapeutic target in osteoarthritis, rheumatoid arthritis and certain cancers ${ }^{25}$. The attenuation of TACE activity in vivo by use of small-interfering RNA (siRNA) ${ }^{26}$, targeted deletion ${ }^{27}$ or pharmacological inhibition ${ }^{28}$ has improved cardiac hypertrophy and the survival rates during endotoxic shock and pneumococcal meningitis. Notably, administration of TACE and MMP inhibitors to humans in clinical trials has proven to effectively decrease inflammatory mediators ${ }^{29}$ and diminish disease activity ${ }^{30}$.

We have already documented that there are a multitude of mechanisms by which ADAM28 may promote inflammation and ultimately metabolic dysfunction. In addition to its proteinase functions, the disintegrin domain of ADAM28 also plays an important role in inflammation. The disintegrin domain may serve as a ligand for the integrin alpha(4) beta(1) which is expressed on a number of leukocyte populations including macrophages which are a major source of the pro-inflammatory 
cytokine TNF- $\alpha^{31}$. The implications of the interaction of ADAM28 with alpha(4) beta(1) are twofold: (i) it may promote inflammation due to the accumulation of lymphocytes with other leukocyte populations and (ii) secondly, it may function to target the active protease to substrates at the site of cell-cell contact. Further supporting this concept of ADAM28 in inflammation, we have recently elegantly demonstrated that the disintegrin-like domain of ADAM28 binds the extracellular portion of P-selectin glycoprotein ligand-1 (PSGL-1) on leukocytes ${ }^{32}$. This enhances PSGL-1/P-selectinmediated cell adhesion to endothelial cells, promoting leukocyte rolling and adhesion to blood vessel endothelial cells and subsequent migration into tissues, therefore facilitating inflammation.

We have previously illustrated that one of the major substrates of ADAM28 is IGFBP- $3^{17}$. This finding may have major clinical relevance as IGFBP-3 has been implicated in the enhancement of adipogenesis $^{33}$. Furthermore, mice over expressing IGFBP-3 showed increased epididymal fat pad $\operatorname{size}^{34}$. This trait is believed to be due to the elevated insulin-like growth factor (IGF-1) levels in the transgenic mice. Using over-expression systems and cells in which ADAM28 and TNF- $\alpha$ are expressed endogenously, we have shown in our current study that human ADAM28 is a novel sheddase for human TNF- $\alpha$. Using molecular and pharmacological techniques, we highlighted that a direct consequence of ADAM28 inhibition is decreased cleavage of cell membrane bound TNF- $\alpha$. Hence, based on our results it is plausible that therapeutic ADAM28 inhibition may reduce inflammation due to diminished TNF- $\alpha$ shedding and binding of its disintegrin domain to integrins and PSGL-1. Worley and colleagues ${ }^{35}$ aimed to ascertain whether treatment of human monocytes with low density lipoprotein (LDL) from type 2 diabetics (T2D) would alter metalloproteinase expression. They discovered that LDL from T2D patients significantly increased mRNA levels for ADAM17, ADAM15 and importantly, ADAM28. This study suggested that the LDL-induced increases in metalloproteinase expression may lead to increased release of adhesion molecules and TNF- $\alpha$ release. We have now formally demonstrated the latter in our current study.

Our project is most significant as it has demonstrated for the first time, the importance of ADAM28 in the metabolic syndrome and further supports that metalloproteinase inhibition is a potential therapeutic target for anti-obesity agents. Importantly, there is the potential for ADAM28 mediated TNF- $\alpha$ cleavage to be implicated in numerous other immunopathological conditions.

\section{METHODS}

Human microarray for the identification of metalloproteinases involved in obesity and T2D. 
RNA samples (from peripheral blood mononuclear cells) were obtained from the San Antonio Family Heart Study (SAFHS), a study of risk factors for cardiovascular disease in Mexican Americans living in and around San Antonio, Texas ${ }^{36}$. The SAFHS is a large family-based genetic epidemiological study including 1431 individuals from 42 extended families at baseline. Individuals from large randomly selected, multigenerational pedigrees were sampled independent of their phenotype or the presence or absence of disease. All participants in the SAFHS provided informed consent. The study and all protocols were approved by the Institutional Review Board at the University of Texas Health Science Centre at San Antonio (San Antonio, TX).

\section{Cell Culture experiments.}

L6 myoblast cells, HEK293 and THP-1 cells ( $<6$ passages) were purchased from the American Type Culture Collection (Manassas, VA). Cells were cultured at $37^{\circ} \mathrm{C}, 5 \% \mathrm{CO}_{2}$ in a humidified chamber. L6 myoblasts were seeded in 6 well culture plates and grown in DMEM (low glucose; Gibco $)+10 \%$ FBS $+1 \%$ penicillin/streptomycin. Differentiation of the myoblasts was induced by transferring cells to medium containing $2 \%$ fetal calf serum when the myoblasts were $\sim 90 \%$ confluent. Experimental treatments commenced after 7 days of differentiation when nearly all myoblasts had fused to form myotubes. Upon the day of experimentation, cells were serum starved for 4 hrs and treated with cell-free HEK293 conditioned media for 48hrs. HEK293 cells were cultured in DMEM (low glucose; Gibco) $+10 \%$ FCS and 1\% penicillin/streptomycin. THP-1 human monocytes were cultured in DMEM (low glucose; Gibco) $+5 \%$ FCS and $1 \%$ penicillin/streptomycin. To differentiate the THP-1 cells to macrophages, when cells were confluent, $5 \mathrm{ng} / \mathrm{mL}$ of PMA was added and cell free supernantant was collected after $48 \mathrm{hrs}^{37}$. In other experiments, undifferentiated THP-1 cells were stimulated with $10 \mathrm{ng} / \mathrm{mL}$ recombinant TNF- $\alpha$

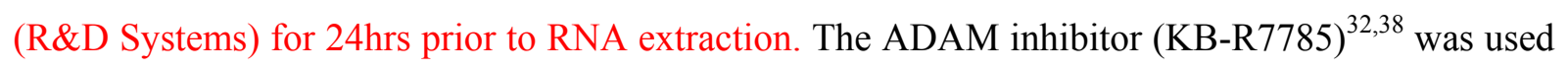
at $1 \mu \mathrm{M}$ for HEK293 cells and 5 $\mu \mathrm{M}$ for THP-1 cells to inhibit ADAM28 activity.

\section{Immunoprecipitation.}

Twenty four hrs post transfection, HEK293 cells were washed with ice-cold PBS and then lysed with immunoprecipitation buffer (50mM Tris, 130mM NaCl, 5mM EDTA, 1\% NP-40, 1mM PMSF, $1 \mathrm{mM} \mathrm{NaF}$ ) containing protease and phosphatase inhibitors (Roche). After protein determination, $200 \mu \mathrm{g}$ of protein was cleared with a mixture of washed protein A/protein G (1:1) sepharose beads (Roche) for $1 \mathrm{hr}$. After centrifugation, the protein solution was added to freshly washed sepharose beads and mouse anti-human ADAM28 antibody $(297-2 \mathrm{~F} 3 ; 3 \mu \mathrm{g} / \mathrm{mL})$ and incubated $\mathrm{O} / \mathrm{N}$ at $4^{\circ} \mathrm{C}$ with mixing. The following day, the beads were washed 3 times with ice-cold PBS and then $40 \mu \mathrm{L}$ 
of laemmeli sample buffer was added and boiled for $10 \mathrm{~min}$. The supernatant was then subjected to immunoblotting for human pro-TNF- $\alpha(27 \mathrm{kD})$.

\section{Transfections}

HEK293 cells were seeded into 6 well cell culture dishes and transiently transfected with either empty pCMV-Tag4a vector or vectors containing the cDNA for human proADAM $28^{38}$ or TNF- $\alpha$ using Lipofectamine reagent (Life Technologies). Cell free culture supernatants were collected after 48hrs. Human TNF- $\alpha$ in the cell-free culture supernatant and ADAM28 in the cell lysate collected from transfected HEK293 cells were measured using commercially available enzyme linked immunosorbent assay kits [(TNF- $\alpha$; R\&D Systems, DY210) and (ADAM28; USCN LifeSciences Inc, E99202Hu). In all siRNA experiments, HEK293 and THP1 cells were seeded into 12 well cell culture dishes and transfections were conducted using X-tremeGENE HP DNA transfection reagent (Roche) and incubated for $48 \mathrm{hrs}$. THP1 cells were stimulated with $1 \mu \mathrm{g} / \mathrm{ml}$ lipopolysaccharide for the duration of the transfection. A Cy3 DS transfection control (scrambled siRNA; IDT) was used at $10 \mathrm{nM}$ as a negative control. ADAM28 was knocked down using Silencer Select siRNA at 10nM (A28 siRNA 1= s21321; A28 siRNA 2=s21323; A28 siRNA 3=s21322; Applied Biosystems).

\section{Immunofluorescence.}

Cells were fixed with ice-cold methanol:acetone and were pre-incubated in $0.3 \% \mathrm{H}_{2} \mathrm{O}_{2}$ to quench endogenous peroxidase, followed by incubation in 10\% FCS to block non-specific background staining. Sections were incubated overnight at $4^{\circ} \mathrm{C}$ with rabbit anti-human TNF- $\alpha$ antibody diluted 1:100 (Cell Signalling Technologies, Beverly, MA; \#3707) or mouse monoclonal anti-human ADAM28 (297-2F3) antibody $(10 \mu \mathrm{g} / \mathrm{mL})^{32,38}$. It should be noted that rabbit and mouse IgG isotype control antibodies were used as negative controls (BD Biosciences). The TNF- $\alpha$ primary antibody was detected with anti-rabbit 488 (Invitrogen; A11034) and the ADAM28 antibody was detected with either anti-mouse 546 (Invitrogen; A11003) or anti-mouse 488 (Invitrogen; A11029) for 30 min at room temperature. Nuclei were stained with DAPI (Sigma; D9542) and then cells were visualised using an Olympus IX71 microscope.

\section{Determination of gene and protein expression.}

RNA was extracted using Trizol reagent (Invitrogen) and cDNA synthesis was performed using the High Capacity RNA-to-cDNA kit (Applied Biosystems). Real-time PCR to determine the mRNA abundance of human ADAM28 and 18S (house-keeper gene) was performed using a Rotor-gene real-time PCR machine (Qiagen) using pre-developed TaqMan probe (FAM labelled) and primer 
sets for human ADAM28 (Hs00248020_m1) and human 18S (Hs03928990_g1) from Applied Biosystems. Quantitation was conducted as previously described ${ }^{39}$.

For western blotting analyses, cells were washed in ice-cold PBS and then lysed using cytosolic extraction buffer (10mM HEPES; $3 \mathrm{mM} \mathrm{MgCl}_{2} ; 14 \mathrm{mM} \mathrm{KCl} ; 5 \%$ glycerol; $0.2 \%$ IPEGAL) containing phosphatase and protease inhibitors (Roche). Cell lysates were cleared and protein concentration calculated using protein assay solution (Biorad). Protein lysates were solubilized in Laemmeli sample buffer and boiled for $10 \mathrm{~min}$, resolved by SDS-PAGE on 10\% polyacrylamide gels, transferred by semi-dry transfer to PVDF membrane, and then blocked with 5\% milk powder. Membranes were then incubated overnight at $4^{\circ} \mathrm{C}$ in Odyssey blocking buffer containing either primary antibody against phospho JNK (Thr183/Tyr185) (Cell Signaling, Beverly, MA, \#4668), total JNK (Cell Signalling, Beverly, MA; \#9252), TNF- $\alpha$ (Cell Signalling, Beverly, MA; \#3707), $\beta$-actin (Ab-Cam; ab6276) or human ADAM28 antibody $(297-2 \mathrm{~F} 3)(1 \mu \mathrm{g} / \mathrm{mL})^{32,38}$ using recommended dilutions. Membranes were washed 3 times in washing buffer and incubated for 60 min at room temperature with either IRDye 680LT conjugated goat anti-mouse IgG, IRDye 800CW conjugated goat anti-mouse IgG or IRDye $800 \mathrm{CW}$ conjugated goat anti-rabbit IgG secondary antibodies (LI-COR Biosciences, Lincoln, Nebraska, USA) at a 1:10,000-20,000 dilution, followed by 3 times 5 min washes in washing buffer. The protein bands were detected and quantified using the Odyssey infrared imaging system (LI-COR Biosciences, Lincoln, Nebraska, USA).

\section{Statistics}

Associations between ADAM28 lymphocyte-derived gene expression and clinical parameters in the Mexican American pedigrees were determined using a linear model allowing for residual nonindependence amongst family members as a function of their genetic relationships. A likelihood ratio statistic was utilized to test whether ADAM28 expression significantly predicted a relevant standard clinical parameter (fasting insulin, BMI, HDL-C, relative fat mass, and the HOMA-IR index of insulin resistance). Other primary covariates were simultaneously controlled for including sex and age. All analyses were performed using the statistical package, SOLAR.

In the cell culture experiments all data were analysed from 3 independent experiments and paired ttests were used to statistically analyse the data where appropriate.

\section{ACKNOWLEDGEMENTS}


This study was supported by grants from the Diabetes Australia Research Trust (DART) and the Medical Research Foundation (Royal Perth Hospital). VBM and JJ are supported by National Health and Medical Research Council Career Development fellowships. We are grateful to the participants of the San Antonio Family Heart Study. Data collection for the SAFHS was supported by a grant from the National Institute for Heart, Lungs and Blood (HL045222). A generous donation from the Azar/Shepperd families paid for the transcriptional profiles. SOLAR is supported by the National Institute for Mental Health (MH059490). Parts of this investigation were conducted in facilities constructed with support from the Research Facilities Improvement Program Grant Number C06 RR013556 from the National Center for Research Resources, National Institutes of Health. The AT\&T Genomics Computing Center supercomputing facilities used for this work were supported in part by a gift from the AT\&T Foundation and with support from the National Center for Research Resources Grant Number S10 RR029392. We thank Professor Stefan Rose-John (Kiel University) for providing the human TNF- $\alpha$ expression vector and Richelle Searles for technical assistance.

\section{Figure Legends}

Figure 1. Human ADAM28 and human TNF- $\alpha$ co-localise and co-immunoprecipitate in transfected HEK293 cells. Double staining for ADAM28 (A); TNF- $\alpha$ (B) and both proteins merged (C). Bar $=10 \mu \mathrm{m}$. ADAM28 co-immunoprecipitated with TNF- $\alpha$ in double transfected cells (D).

Figure 2. Human ADAM28 sheds human TNF- $\alpha$ in transfected HEK293 cells. TNF- $\alpha$ cleavage was promoted with ADAM28 co-transfection. Two micrograms of each plasmid was transfected and empty vector was used when ADAM28 or TNF- $\alpha$ were alone (A). The ADAM28 inhibitor, KBR7785 was used at $1 \mu \mathrm{M}$ for $48 \mathrm{hrs}$ and TNF- $\alpha$ cleavage was measured by ELISA (B) or immunoblotting (C). Data expressed as mean \pm SEM. ${ }^{*} \mathrm{p}<0.05$ for indicated differences; $\mathrm{n}=3-6$ from at least 2 independent experiments.

Figure 3. Molecular knock-down of human ADAM28 protein levels diminishes TNF- $\alpha$ shedding in HEK293 cells. HEK293 cells were co-transfected with TNF- $\alpha$ and ADAM28 vectors in addition to either scrambled siRNA or three different human ADAM28 (A28) siRNA. After 48hrs, ADAM28 mRNA (A), ADAM28 protein (B, C and D), TNF- $\alpha$ shedding (E; top panel) and membrane bound pro-TNF- $\alpha$ protein levels (E; bottom panel) were determined. Data expressed as mean $\pm \operatorname{SEM}(\mathrm{A}, \mathrm{C}, \mathrm{D}$ and $\mathrm{E}) .{ }^{*} \mathrm{p}<0.05$ compared to scrambled siRNA (scrambled); $\mathrm{n}=3-6$ from at least 2 independent experiments. 
Figure 4. ADAM28 shed TNF- $\alpha$ promotes activation of stress kinases in HEK293 and L6 skeletal muscle cells. HEK293 cells were transfected with TNF- $\alpha$ vector, ADAM28 vector or cotransfected and phosphorylation of JNK was measured (A and B). L6 myotubes were treated with conditioned media from HEK293 cells transfected with TNF- $\alpha$ vector (TNF- $\alpha$ ) or TNF- $\alpha$ and ADAM28 vectors for $48 \mathrm{hrs}$ and then examined for phosphorylation of JNK (C and D). Data expressed as mean \pm SEM (B and D). ${ }^{*} \mathrm{p}<0.05$ for indicated differences; $\mathrm{n}=3-6$ from at least 2 independent experiments.

Figure 5. TNF- $\alpha$ stimulation promotes expression of ADAM28 in HEK293 cells and THP1 monocytes. Cells were transfected with TNF- $\alpha$ vector, ADAM28 vector or co-transfected and cellular ADAM28 levels were then measured by ELISA (A). THP1 cells were stimulated with $10 \mathrm{ng} / \mathrm{mL}$ rhTNF- $\alpha$ for $24 \mathrm{hrs}$ and ADAM28 mRNA levels were assessed (B). Data expressed as mean \pm SEM. ${ }^{*} p<0.05$ for indicated differences; $n=3-6$ from at least 2 independent experiments.

Figure 6. Inhibition of endogenous ADAM28 activity inhibits TNF- $\alpha$ shedding in human PMA-differentiated and LPS stimulated THP-1 monocytes. ADAM28 expression (green) after PMA induced differentiation of THP1 cells. Nuclei are stained blue with DAPI (A), inhibition of ADAM28 activity with KBR7785 $(5 \mu \mathrm{M})$ decreases PMA-induced TNF- $\alpha$ shedding (B) and inhibition of ADAM28 with siRNA reduces LPS stimulated TNF- $\alpha$ shedding (C). Data expressed as mean \pm SEM $\left(B\right.$ and $C$ ). ${ }^{*} p<0.05$ for indicated differences; $n=3-6$ from at least 2 independent experiments; Bar $=10 \mu \mathrm{m}$. 


\section{REFERENCES}

1) Flegal KM, Carroll MD, Ogden CL, Johnson CL. Prevalence and trends in obesity among US adults, 1999-2000. JAMA 2002; 288: 1723-1727.

2) Hirosumi J, Tuncman G, Chang L, Gorgun CZ, Teoman Uysai K, Maeda K et al. A central role for JNK in obesity and insulin resistance. Nature 2002; 420: 333-336.

3) Chiang S-H, Bazuine M, Lumeng CN, Galetka LM, Mowers J, White NM et al. The protein kinase IKKع regulates energy balance in obese mice. Cell 2009; 138: 961-975.

4) Arkan MC, Hevener AL, Greten FR, Maeda S, Li Z-W, Long JM et al. (2005) IKK- $\beta$ links inflammation to obesity-induced insulin resistance. Nat Med 2005; 11: 191-198.

5) Delibegovic M, Zimmer D, Kauffman C, Rak K, Hong E-G, Cho Y-R et al. Liver-specific deletion of protein-tyrosine phosphatase 1B (PTP1B) improves metabolic syndrome and attenuates diet-induced endoplasmic reticulum stress. Diabetes 2009; 58: 590-599.

6) Solinas G, Vilcu C, Neels JG, Bandyopadhyay GK, Luo J-L, Naugler W et al. JNK1 in hematopoietically derived cells contributes to diet-induced inflammation and insulin resistance without affecting obesity. Cell Metab 2007; 6: 386-397 .

7) Carey AL, Steinberg GR, Macaulay SL, Thomas WG, Holmes AG, Ramm G et al. Interleukin-6 increases insulin-stimulated glucose uptake and fatty acid oxidation in vitro via AMP-activated protein kinase. Diabetes 2006; 55: 2688-2697.

8) Reed JA, Clegg DJ, Blake Smith K, Tolod-Richer EG, Matter EK, Picard LS et al. GM-CSF action in the CNS decreases food intake and body weight. J Clin Invest 2005; 115: 3035-3044.

9) Fruebis J, Tsu-Shuen T, Javorschi S, Ebbets-Reed D, Erickson MRS, Yen FT et al. Proteolytic cleavage product of $30-\mathrm{kDa}$ adipocyte complement-related protein increases fatty acid oxidation in muscle and causes weight loss in mice. Proc Natl Acad Sci USA 2001; 98: 2005-2010.

10) Pelleymounter MA, Cullen MJ, Baker MB, Hecht R, Winters D, Boone T et al. Effects of the obese gene product on body weight regulation in ob/ob mice. Science 1995; 269: 540-543.

11) Hong EG, Ko HJ, Cho YR, Kim HJ, Ma Z, Yu TY et al. Interleukin-10 prevents diet-induced insulin resistance by attenuating macrophage and cytokine response in skeletal muscle. Diabetes 2009; 58: 2525-2535.

12) Steinberg GR, Michell BJ, van Denderen BJ, Watt MJ, Carey AL, Fam BC et al. Tumor necrosis factor alpha-induced skeletal muscle insulin resistance involves suppression of AMPkinase signaling. Cell Metab 2006; 4: 465-474.

13) Hotamisligil GS, Shargill NS, Spiegelman BM. Adipose expression of tumor necrosis factor- $\alpha$ : direct role in obesity-linked insulin resistance. Science 1993; 259: 87-91. 
14) Hotamisligil GS, Arner P, Caro JF, Atkinson RL, Spiegelman BM. Increased adipose tissue expression of tumor necrosis factor- $\alpha$ in human obesity and insulin resistance. J Clin Invest 1995; 95: 2409-2415.

15) Krogh-Madsen R, Plomgaard P, Moller K, Mittendorfer B, Pedersen BK. Influence of TNF- $\alpha$ and IL-6 infusions on insulin sensitivity and expression of IL-18 in humans. Am J Physiol Endocrinol Metab 2006; 291: 108-114.

16) Lo JC, Wang Y, Tumanov AV, Bamji M, Yao Z, Reardon CA et al. Lymphotoxin $\beta$ receptordependent control of lipid homeostasis. Science 2007; 316: 285-288.

17) Mochizuki S, Shimoda M, Shiomi T, Fujii Y, Okada Y. ADAM28 is activated by MMP-7 (matrilysin-1) and cleaves insulin-like growth factor binding protein-3. Biochem Biophys Res Commun 2004; 315: 79-84.

18) Mochizuki S, Tanaka R, Shimoda M, Onuma J, Fujii Y, Jinno H, Okada Y. Connective tissue growth factor is a substrate of ADAM28. Biochem Biophys Res Commun 2010; 402: 651-657.

19) Hikichi Y, Yoshimura K, Takigawa M. All-trans retinoic acid-induced ADAM 28 degrades proteoglycans in human chondrocytes. Biochem Biophys Res Commun 2009; 386: 294-299.

20) Howard L, Zheng Y, Horrocks M, Maciewicz RA, Blobel C. Catalytic activity of ADAM28. Febs Lett 2001; 498: 82-86.

21) Fourie, AM, Coles F, Moreno V, Karlsson L. Catalytic activity of ADAM8, ADAM15, and MDC-L (ADAM28) on synthetic peptide substrates and in ectodomain cleavage of CD23. J Biol Chem 2003; 278: 30469-77.

22) Serino M, Menghini R, Fiorentino L, Amoruso R, Mauriello A, Lauro D et al. Mice heterozygous for tumor necrosis factor- $\alpha$ converting enzyme are protected from obesity-induced insulin resistance and diabetes. Diabetes 2007; 56: 2541-2546.

23) Fiorentino L, Vivanti A, Cavalera M, Marzano V, Ronci M, Fabrizi M et al. Increased tumour necrosis factor- $\alpha$ converting enzyme activity induces insulin reistance and hepatosteatosis in mice. Hepatology 2010; 51: 103-110.

24) Reddy ABM, Ramana KV, Srivastava S, Bhatnagar A, Srivastava SK. Aldose reductase regulates high glucose-induced ectodomain shedding of tumor necrosis factor (TNF)- $\alpha$ via protein kinase $\mathrm{C}-\delta$ and TNF- $\alpha$ converting enzyme in vascular smooth muscle cells. Endocrinology 2009; 150: $63-74$.

25) Burrage PS, Brinckerhoff CE. Molecular targets in osteoarthritis: metalloproteinases and their inhibitors. Curr Drug Targets 2007; 8: 293-303. 
26) Wang X, Oka T, Chow FL, Cooper SB, Odenbach J, Lopaschuk GD et al. Tumor necrosis factor- $\alpha$-converting enzyme is a key regulator of agonist-induced cardiac hypertrophy and fibrosis. Hypertension 2009; 54: 575-582.

27) Horiuchi K, Kimura T, Miyamoto T, Takaishi H, Okada Y, Toyama Y et al. TNF- $\alpha$-converting enzyme (TACE/ADAM17) inactivation in mouse myeloid cells prevents lethality from endotoxin shock. J Immunol 2007; 179: 2686-2689.

28) Echchannaoui H, Leib SL, Neumann U, Landmann RMA. Adjuvant TACE inhibitor treatment improves the outcome of TLR2-/- mice with experimental pneumococcal meningitis. BMC Infect Dis 2007; 7: 25-33.

29) Qian M, Bai SA, Brogdon B, Wu J-T, Liu R-Q, Covington MB et al. Pharmacokinetics and pharmacodynamics of DPC 333 ((2R)-2-((3R)-3-Amino-3 44-[2-methyl-4-quinolinyl) methoxy] phenyl\}-2-oxopyrrolidinyl)-N-hydroxy-4-methylpentanamide)), a potent and selective inhibitor of tumor necrosis factor $\alpha$-converting enzyme in rodents, dogs, chimpanzees, and humans. Drug Metab Dispos 2007; 35: 1916-1925.

30) Dezube BJ, Krown SE, Lee JY, Bauer KS, Aboulafia DM. Randomised phase II trial of matrix metalloproteinase inhibitor COL-3 in AIDS-related kaposi's sarcoma: an AIDS malignancy consortium study. J Clin Oncol 2006; 24: 1389-1394.

31) Bridges LC, Tani PH, Hanson KR, Roberts CM, Judkins MB, Bowditch RD. The lymphocyte metalloproteinase MDC-L (ADAM 28) is a ligand for the integrin alpha4beta1. J Biol Chem 2002; 277: 3784-3792.

32) Shimoda M, Hashimoto G, Mochizuki S, Ikeda E, Nagai N, Ishida S et al. Binding of ADAM28 to P-selectin glycoprotein ligand-1 enhances P-selectin-mediated leukocyte adhesion to endothelial cells. J Biol Chem 2007; 282: 25864-25874.

33) Grohmann M, Sabin M, Holly J, Shield J, Crowne E, Stewart C. Characterization of differentiated subcutaneous and visceral adipose tissue from children: the influences of TNF-alpha and IGF-1. J Lipid Res 2005; 46: 93-103.

34) Modric T, Silha JV, Shi Z, Gui Y, Suwanichkul A, Durham SK et al. Phenotypic manifestations of insulin-like growth factor-binding protein-3 overexpression in transgenic mice. Endocrinology 2001; 142: 1958-1967.

35) Worley JR, Huges DA, Dozio N, Gavrilovic J, Sampson MJ. Low density lipoprotein from patients with Type 2 diabetes increases expression of monocyte matrix metalloproteinase and ADAM metalloproteinase genes. Cardiovasc Diabetol 2007; 6: 21-25. 
36) Goring HH, Curran JE, Johnson MP, Dyer TD, Charlesworth J, Cole SA et al. Discovery of expression QTLs using large-scale transcriptional profiling in human lymphocytes. Nat Genet 2007;

39: 1208-1216.

37) Worley JR, Baugh MD, Hughes DA, Edwards DR, Hogan A, Sampson MJ et al. Metalloproteinase Expression in PMA-stimulated THP-1 cells: Effects of peroxisome proliferatoractivated receptor-g (PPARg) agonists and 9-cis-retinoic acid. J Biol Chem 2003; 278: 51340-51346. 38) Mitsui Y, Mochizuki S, Kodama T, Shimoda M, Ohtsuka T, Shiomi T et al. ADAM28 is overexpressed in human breast carcinomas: Implications for carcinoma cell proliferation through degradation of insulin-like growth factor binding protein-3. Cancer Res 2006; 66: 9913-9920.

39) Chan MHS, Carey AL, Watt MJ, Febbraio MA. Cytokine gene expression in human skeletal muscle during concentric contraction: evidence that IL-8, like IL-6, is influenced by glycogen availability. Am J Physiol Regul Integr Comp Physiol 2004; 287: 322-327. 
Table 1. Parameters of the metabolic syndrome are associated with high ADAM28 expression in human peripheral blood mononuclear cells.

\begin{tabular}{|c|c|c|}
\hline Parameter & p-value & Direction of correlation \\
\hline Fasting insulin & 0.0033 & Positive \\
\hline BMI & $8.8 \times 10^{-6}$ & Positive \\
\hline HDL cholesterol & 0.0079 & Negative \\
\hline Relative fat & $1.4 \times 10^{-9}$ & Positive \\
\hline HOMA-IR & 0.0024 & Positive \\
\hline
\end{tabular}




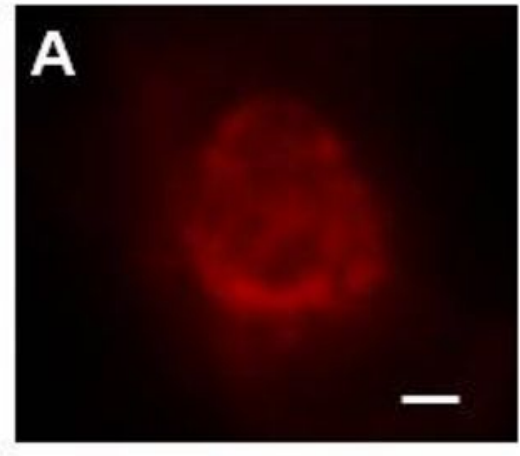

ADAM28

\section{B}

$$
\text { TNF- } \alpha
$$

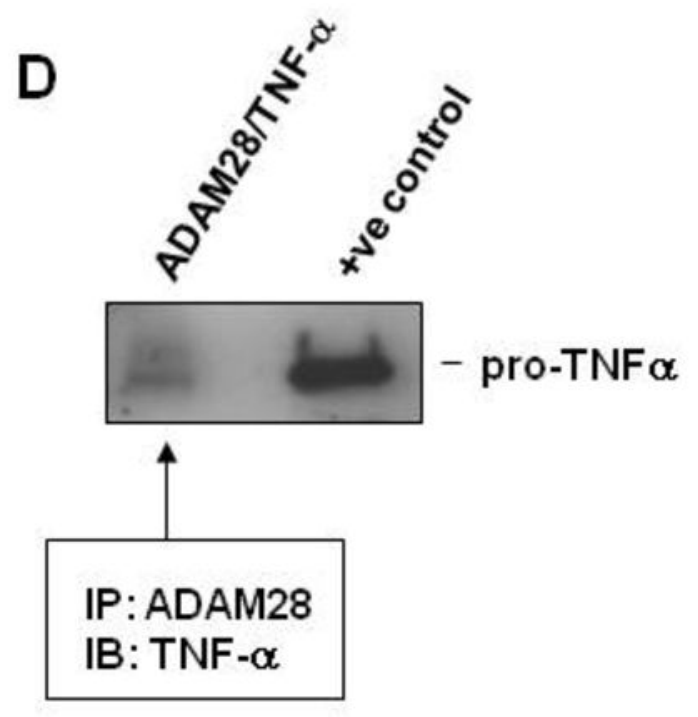

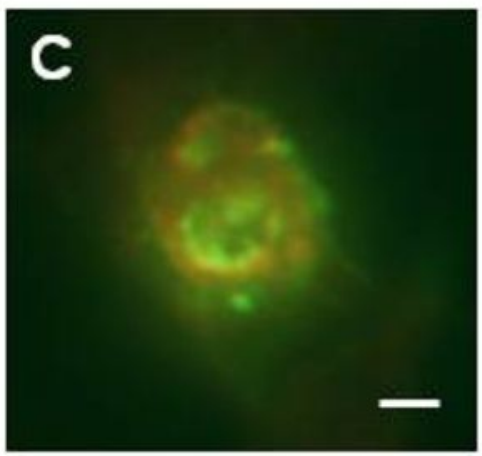

Merge

Figure 1 


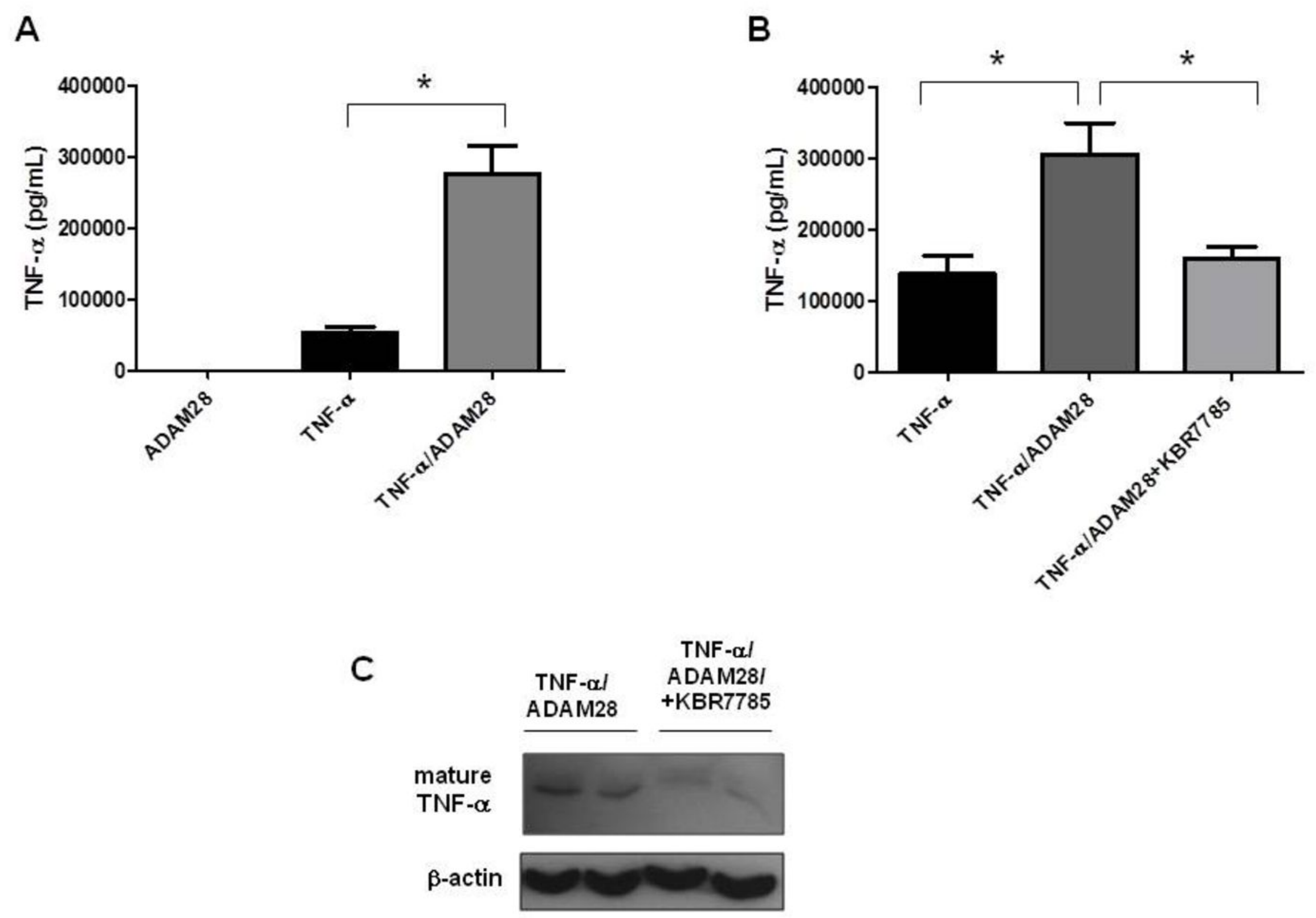

Figure 2 
A

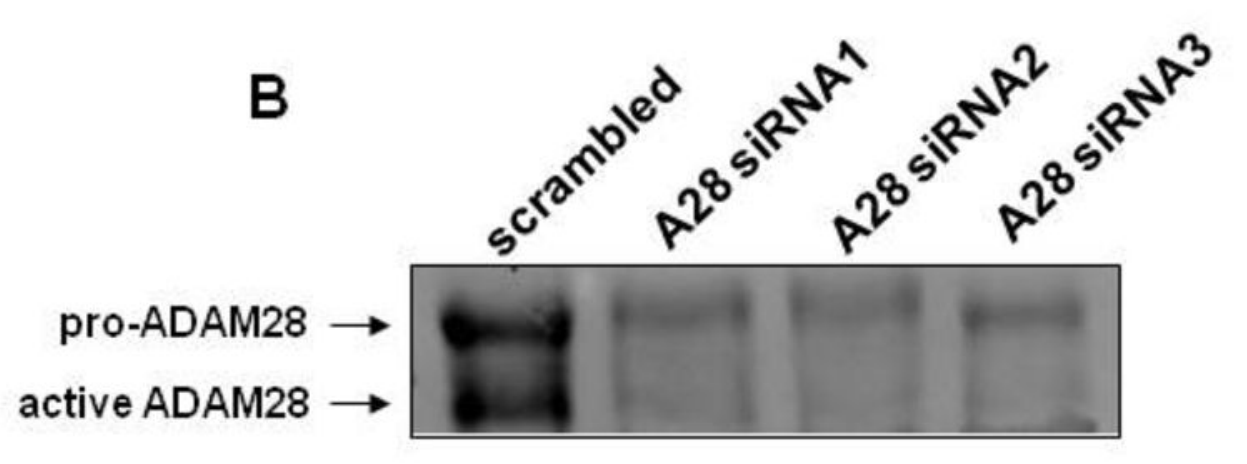

C
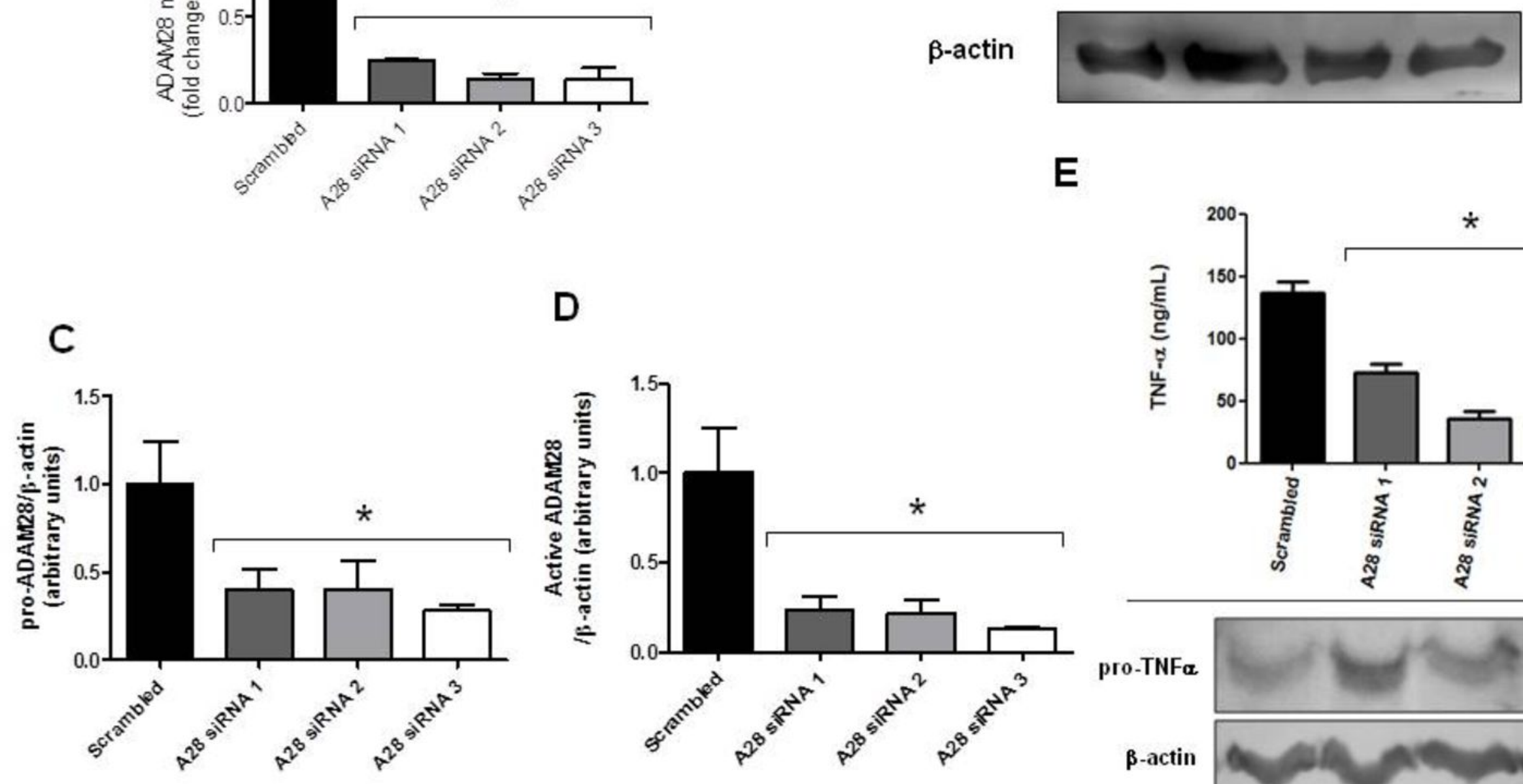

E

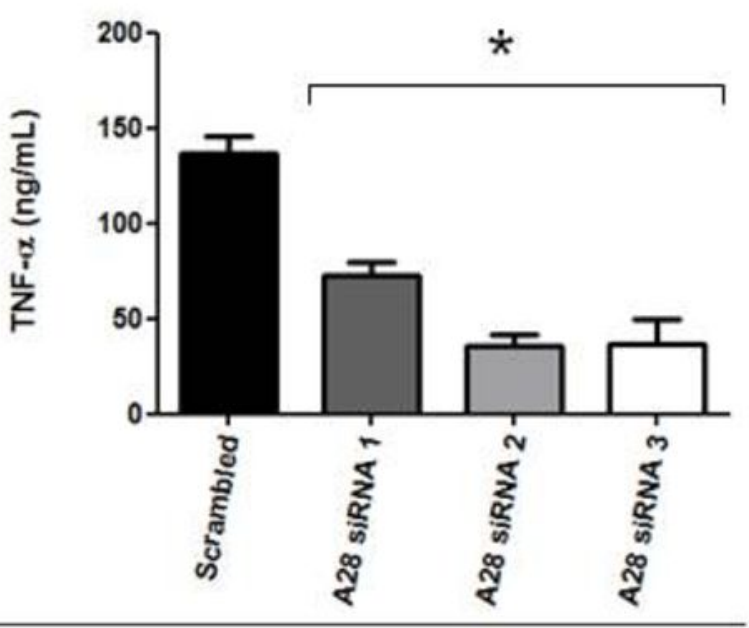

pro-TNFa

$\beta$-actin

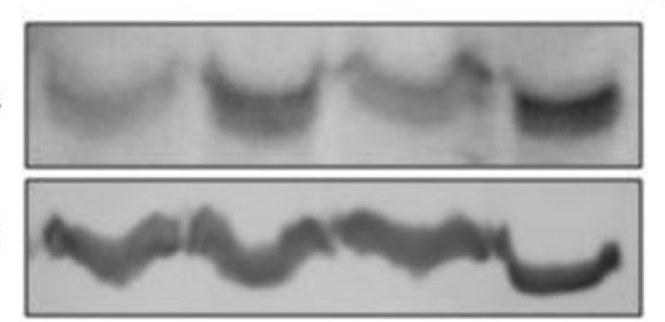

Figure 3 
A

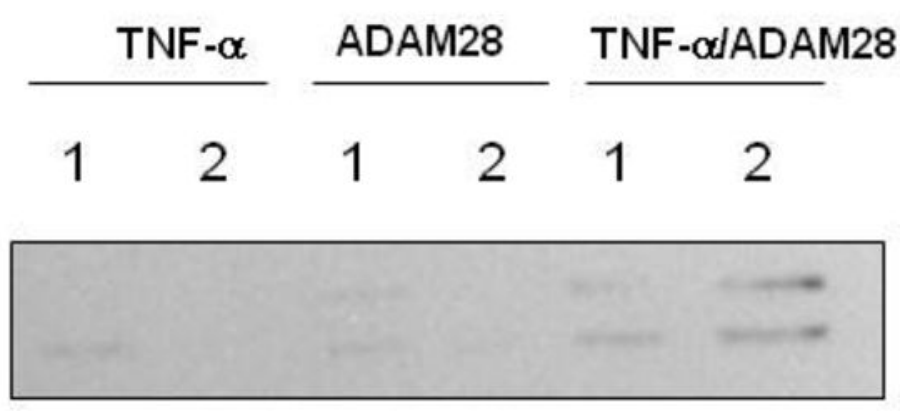

JNK

pJNK 1/2 ll p54-

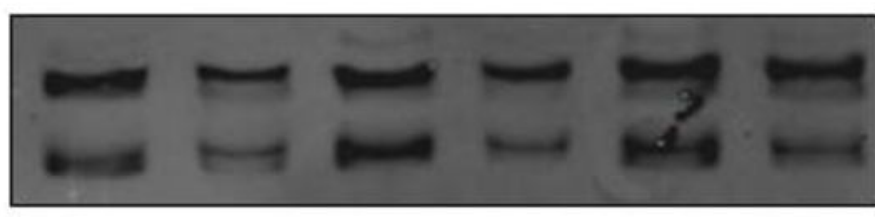

$\boldsymbol{\beta}$-actin
B

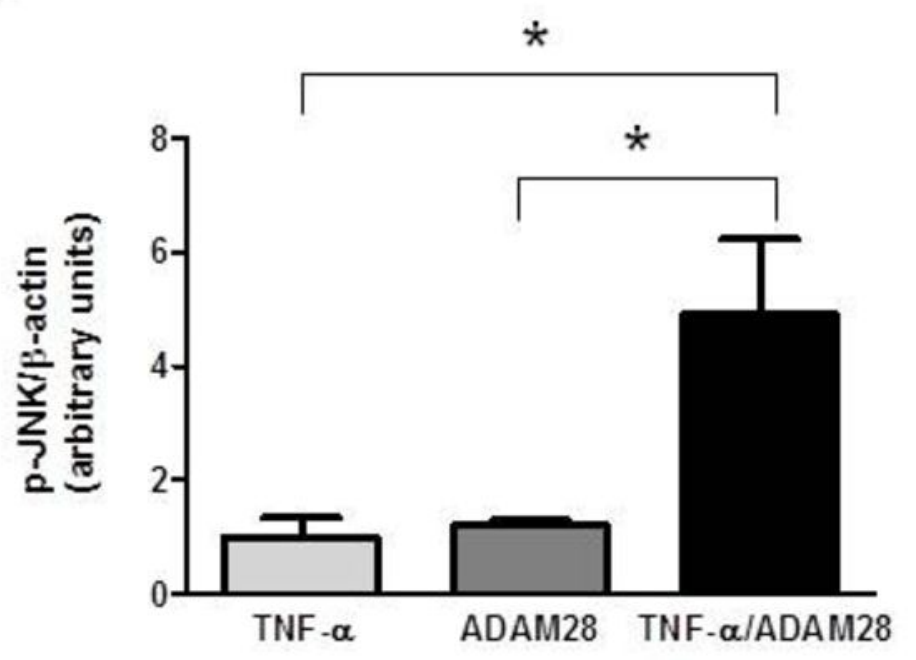

D

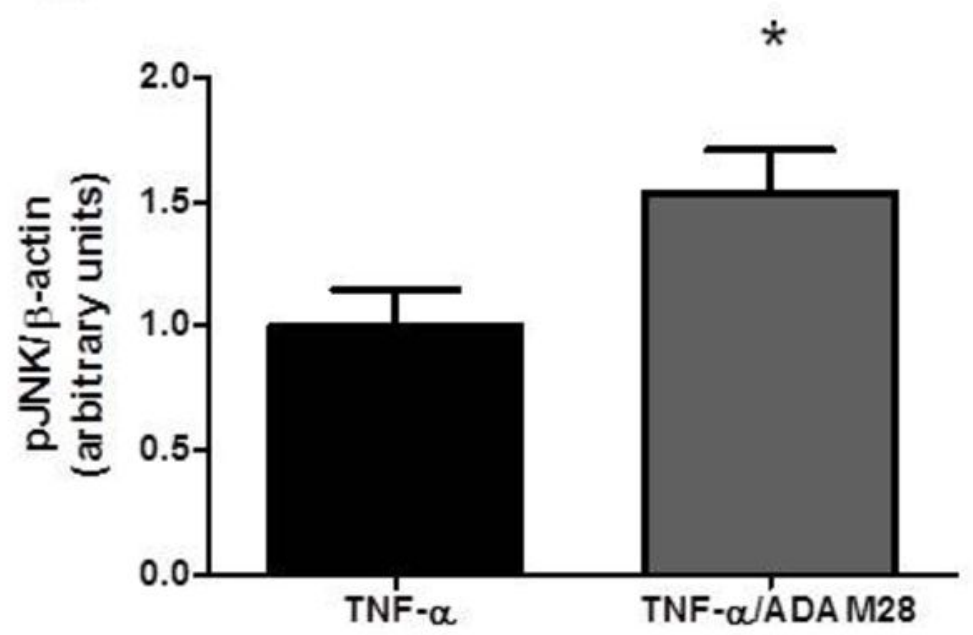

C

$\beta$-actin

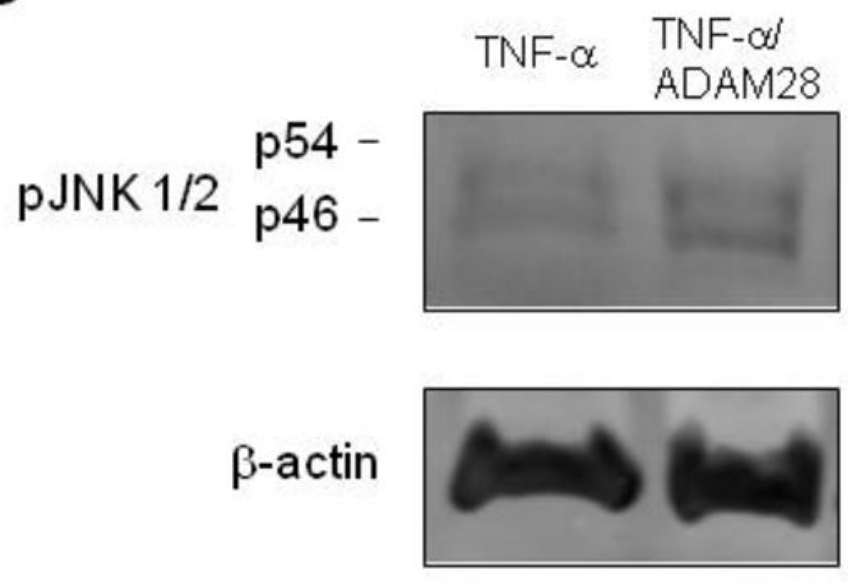

Figure 4 
A

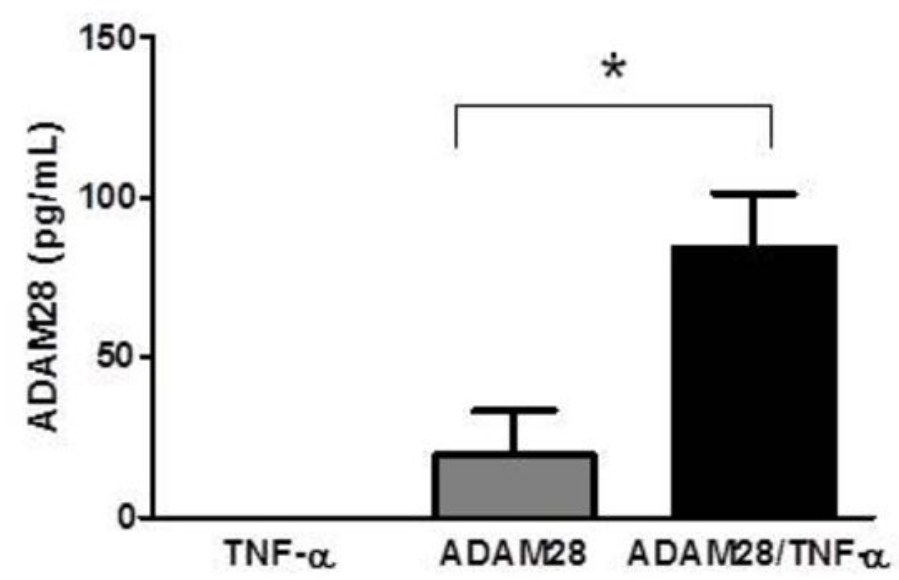

B

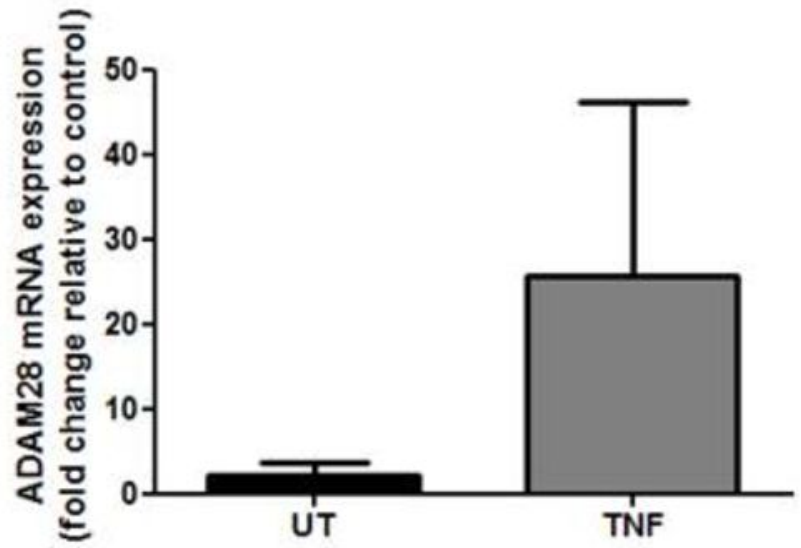

Figure 5 
A

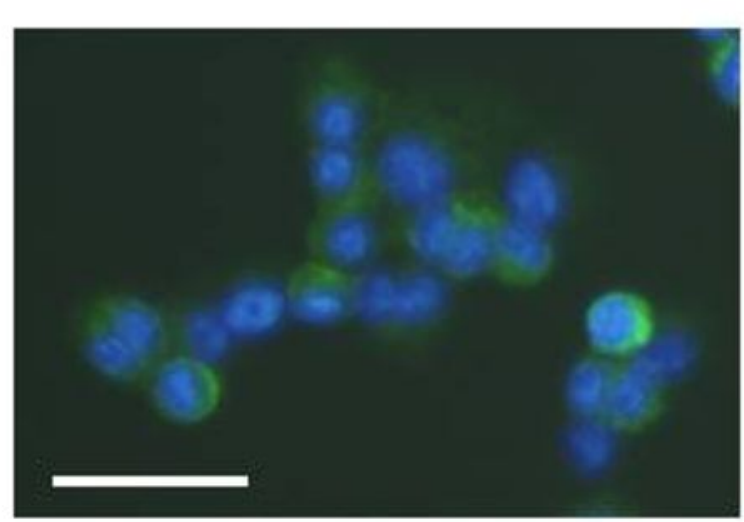

B

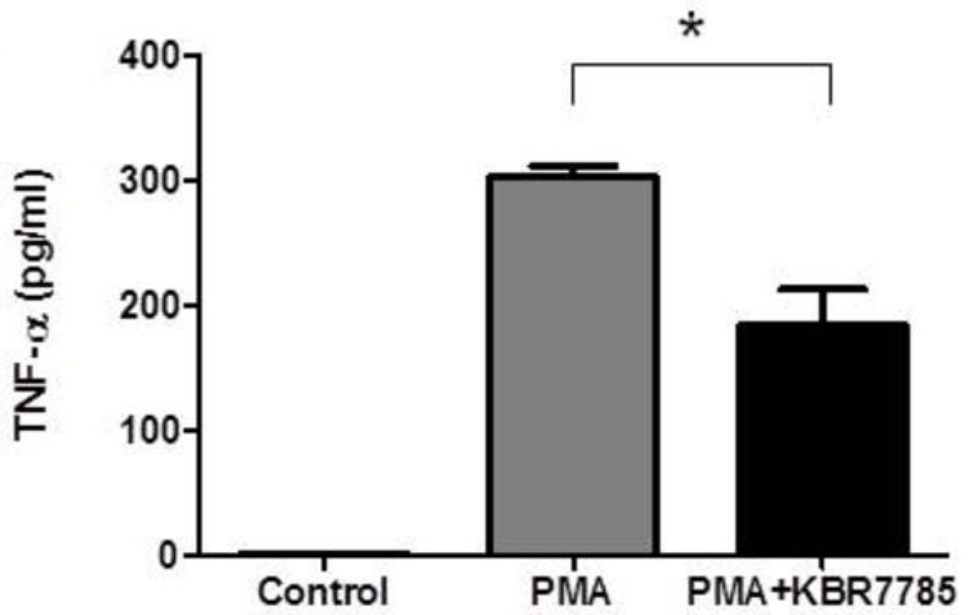

C

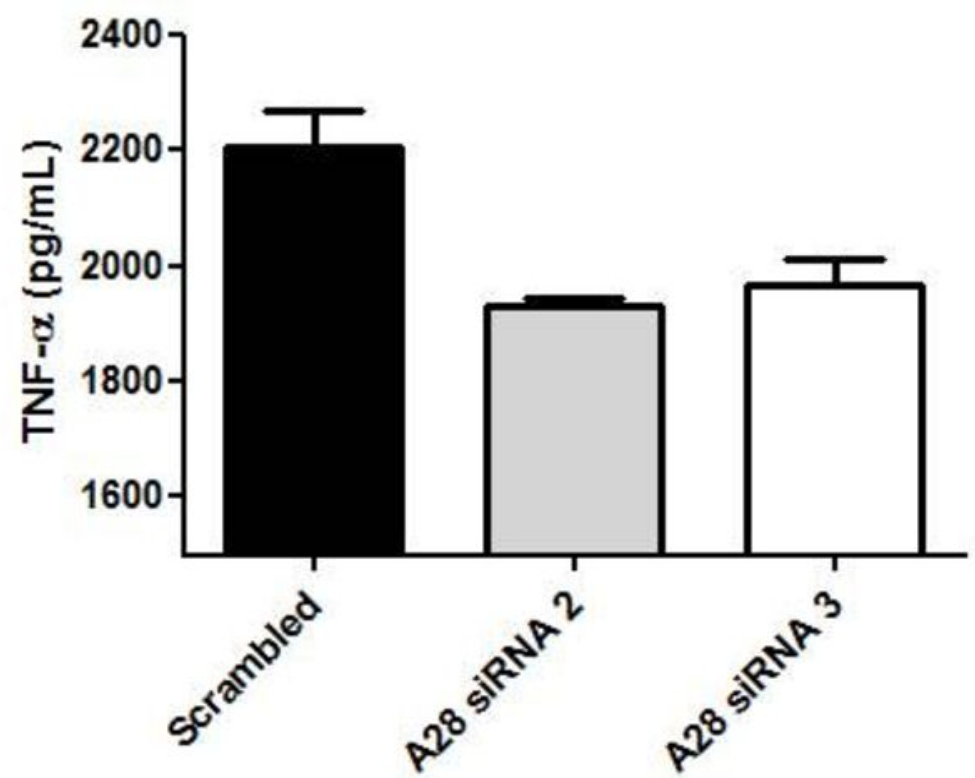

Figure 6 